\title{
HOXA GENE EXPRESSION IMPLICATED IN TREATMENT RESISTANCE AND POOR PROGNOSIS IN GLIOBLASTOMA
}

\author{
Naomi Seidu ${ }^{1}$, Edward Poluyi ${ }^{1}$, Chibuikem Ikwuegbuenyi ${ }^{2}$, Eghosa Morgan ${ }^{3}$ \\ Correspondence: ikwuegbuenyichibuikem@gmail.com \\ ${ }^{1}$ University of Roehampton, London, United Kingdom. \\ ${ }^{2}$ Lagos University Teaching hospital, Lagos Nigeria. \\ ${ }^{3}$ Irrua Specialist Teaching Hospital, Irrua Edo State, Nigeria
}

\begin{abstract}
Article History:
Received: February 13, 2020

Accepted: June 15, 2020

Published: July 1, 2020

\section{Cite this as:}

Seidu N, Poluyi E,

IkwuegbuenyiC, Morgan E. Hoxa

gene expression implicated in treatment resistance and poor prognosis in glioblastoma. Malang Neurology Journal; 2020.6:105-110.

http://dx.doi.org/10.21776/ub.mnj 2020.006.02.8

ABSTRACT

This review brings into view the prognosis attributable to glioblastoma (GBM) and resistance to treatment, surgical interventions and chemotherapy seem ineffective at procuring a better prognosis for patients with the disease. Albeit there exist varying interventions for GBM, the median survival still comes to 12 to 15 months for afflicted patients, this has aroused the need for improvement in treatment success The principal goal is to create a better prognosis and have a decline in treatment resistance invariably leading to better survival rates via adequate treatment for GBM. A relationship exists between HOX genes (homeobox genes) and glioblastoma as is evident from literature. Treatment resistance has been observed in overexpression of HOX genes, the effectiveness of treatment could result from silencing these genes A series of studies have highlighted the role that HOX genes play in glioblastoma prognosis. Promotion of human glioblastoma initiation, aggressiveness, and resistance to Temozolomide has been associated with HOXA9 as shown by Pojo et al. The role of HOX gene expression in cancer stem cells should be studied as it could provide a means of designing CSC-targeted therapies, as CSCs play a part in initiation and progression of solid tumors.
\end{abstract}

Keywords: HOXA Gene- Homeobox genes cluster, GBM- Glioblastoma, temozolomide, signaling pathways, RNA, Wnt.

\section{Introduction}

Glioblastoma (GBM), a type of astrocytic tumor, is a frequent and deadly type of brain tumor, that originate from astrocytes. ${ }^{1} \quad$ Astrocytoma, oligodendrogliomas and ependymomas are other subgroups to these tumors. ${ }^{2}$ Classification of GBM by The world health organization (WHO) is through a series of grades. The first grade is characterized by lesions with a low rate of proliferation, surgical intervention is the usual treatment modality, ${ }^{1}$ and grade two, three, and four, however, are considered highly aggressive and invasive thus, needing more interventions in addition to surgery. Glioblastoma can be considered a rare type of tumor, its incident rate is approximately 10 per 100,00 people, it is a major health issue because of its abysmal prognosis and small rate of survival. ${ }^{3}$ The tumor can occur in any age group with the greatest probability of development between 50 to 60 years, ${ }^{1,4,5}$ a higher incidence is seen more in males than in females. ${ }^{3}$ Glioblastoma lower prevalence is seen in those of African descent, higher incidents are more in Caucasians, Latinos, and Asians. ${ }^{6}$

A huge cause of concern is identifying the carcinogenic causes of malignant growth. ${ }^{1}$ Albeit, exposure to ionizing radiation and harmful substances, are risk factors that have been validated to be attributable to GBM. $^{7}$ There is evidence suggesting that this substance increases the chances of developing glioblastoma.

Salvati et al 2003, found that more than 116 occurrences of glioblastoma are as a direct result of radiation presentation, ${ }^{8}$ these discoveries were likewise reflected in instances of radiation treatment for leukemia. Diet and way of life decisions still can't seem to be related to an expanded danger of glioblastoma. Hereditary hazard factors still can't seem to be affirmed, with only 5 to $10 \%$ of glioblastoma cases having been seen in family cases. ${ }^{9}$ However, links have been made between some diseases, for example, neurofibromatosis and more noteworthy occurrence of glioblastoma. ${ }^{9,10}$ Supratentorial regions of the cerebral hemispheres are the areas were development regularly occurs, ${ }^{1}$ the spinal cord and brainstem could likewise be afflicted. ${ }^{11}$ Growth happens in the white matter and is comprised of a solitary developing and multiplying lesion. ${ }^{12}$ The vascular endothelial cells frequently take on a glomeruloid like structure, different attributes are astrocytoma like with single-core cell and undifferentiated with mitotic characteristics. ${ }^{2,12}$

There is raised intracranial pressure causing mild edema and a move in cerebrum contents, ${ }^{1}$ bringing about 
manifestations with the spontaneous onset, for example, serious headaches accompanied by vomiting; which is one of the prime presentations seen in patients with Glioblastoma. Furthermore, neurocognitive deficiencies could be present, brain cell necrosis which results from the mass with great invasiveness, such deficits include weakened vision or hearing and memory disturbances. Seizures could also be present. ${ }^{13}$ This survey aims at examining HOX qualities and their implications in resistance to treatment and the abysmal prognosis associated with the disease.

\section{Discussion}

Disparity still exists in treatment success in glioblastoma, although there have been advances in treatment modalities, which is in part as a result of features of the disease and localization of the growths, features of which are surrounded around excessive growth. ${ }^{1,14}$ Surgical resection is the primary course of treatment and is usually the first intervention modality that is used, it is majorly dependent on the grade of the tumor and level of localization, due to this there are limited cases of complete tumor removal. ${ }^{6}$ Relapse is almost inevitable in cases were partial removal was carried out, a number as high as $87 \%$ has been observed, it goes without saying that surgical procedures need to be followed by a course of either chemotherapy or radiotherapy. Forms of radiotherapy such as brachytherapy, stereotactic radiosurgery, and hyper-fractionated radiotherapy have been found to be useful in clearing the remaining tumor cells, these however have side effects that a largely undesirable which would include but not limited to neuronal damage induced by radiation and radiation necrosis. ${ }^{1}$ Chemotherapeutic specialists, for example, Temozolomide (TMZ) and carmustine are normally employed. ${ }^{15}$ These substances are anyway profoundly cytotoxic, patients will in general form a protection from the treatment which causes relapse. ${ }^{16}$ By repressing repair of DNA in cells, TMZ brings about apoptosis of the cells, it is the most widely recognized chemotherapeutic agent. ${ }^{17}$ GBM cell lines, oppose the therapeutic advantages of TMZ, for the most part, because of the high methyl guanine methyltransferase, a protein utilized for the repair of DNA. ${ }^{18}$ Very high mortality rates are still the status quo, in spite of new improvements in treatment with 12 to 15 months being the median rate of survival. ${ }^{19}$

There exists various explanations behind the poor prognosis and high recurrence of cases, one of such is treatment resistance, some line of contentions from studies is "cancer stem cell" speculations. There are alterations on a molecular level that can prompt GBM, epidermal development factor receptor quality transformation is a significant hallmark that was found, intensification, and importantly expression. ${ }^{5}$ An increase in the quantity of subatomic examinations, for example. The "undeveloped cell hypothesis" of GBM was placed in play by means of knowledge made accessible by atomic pathogenesis. The hypothesis argues that glioma undeveloped cells exist inside this cell prompting quick proliferation, ${ }^{20}$ because of the pluripotency of these cells, they have been perceived to be engaged with early-stage improvement, subsequently implicated in the oncogenesis of GMB which are known as Homeobox genes.

\section{Homeobox Genes}

A series of the group of genes that are fundamental regulators controlling the scope of cell activities including apoptosis, separation, motility, and angiogenesis, are Homeobox Genes. ${ }^{21}$

A group from the homeobox genes, HOXA, has been implicated in the pathology of glioblastoma, stemming from their contribution to cell differentiation. These genes act as regulatory transcription factors during embryogenesis. ${ }^{22}$ During embryogenesis, the development of the anterior and posterior axis, are attributable to a transcription factor, Homeo-proteins. They can enhance or diminish their target genes. ${ }^{22}$ It has been seen that HOXA genes are either up or down-regulated in GBM, the aggressiveness of the tumor and its location determine the expressiveness, which varies. ${ }^{22}$ Moreover the transcriptional elements of HOXA qualities notwithstanding them upregulated in malignant growth cells, they additionally repress signaling pathways consequently going about as transcriptional repressors. This offers insight into the idea that over and underexpression of these genes is very significant in the comprehension of GBM oncogenesis.

At times, HOX genes have been demonstrated to be oncogenes as demonstrated by previous studies. ${ }^{23}$ It has been seen as evident from a couple of studies the significance of HOX genes in handling resistance to treatment, these genes have an immediate relationship to treatment failure and poor prognosis. ${ }^{24}$

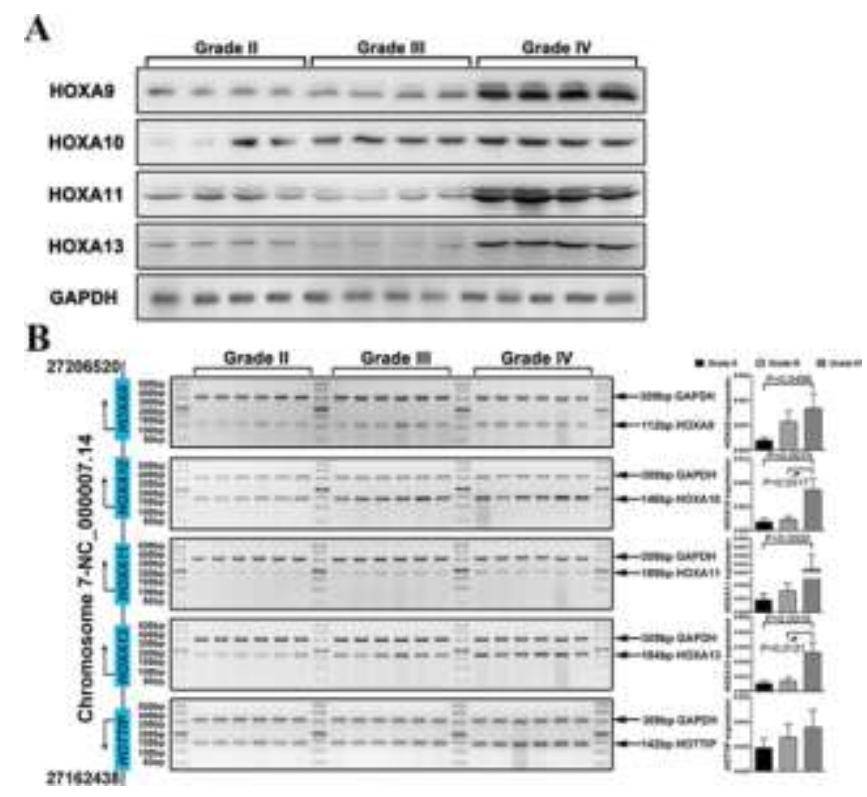

Figure 1. A and B shows western blot analysis found an abnormal upregulation of HOXA9, HOXA10, HOXA11, and HOXA13 in different grade glioma cells and increased HOTTIP transcriptions level. $^{29}$ 

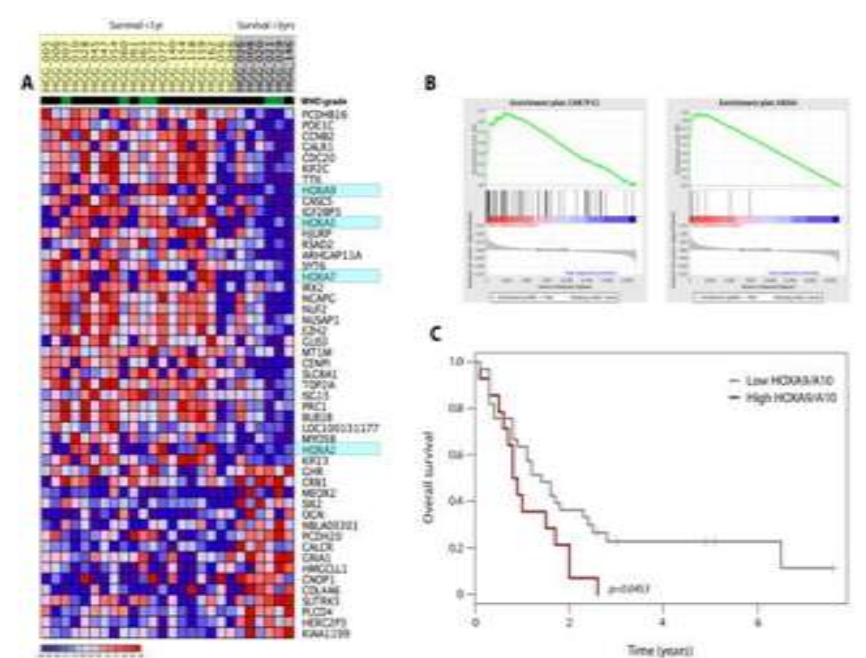

c

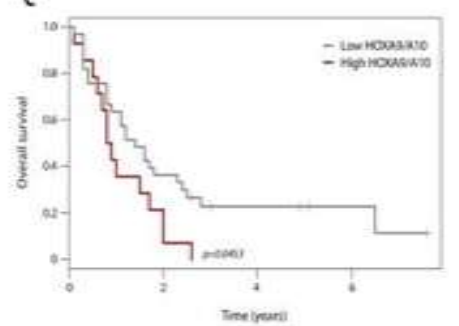

Figure 2. Showing HOXA9 AND HOXA10 as the main genes and resistance to Temozolomide due to their self-renewal signature

The role played by HOX genes in the prognosis of glioblastoma has been featured in a series of studies. Pojo et al contend that HOXA9 drives human glioblastoma initiation, aggressiveness, and protection from Temozolomide. $^{25}$ The HOX genes are important in post developmental regulation and in embryogenesis similarly as earlier stated, ${ }^{26}$ as they are engaged in the differentiation of cells. Changes of any sort to these genes contend Goncalves et al would offer ascent to tumorigenesis in human adults, which we see plainly from the report presented by this investigation that HOXA9 regulation is basic for the reduction in tumor and viability of treatment. ${ }^{27}$ Four cell lines were obtained from the American Type Culture Collection, two human glioblastoma cells U87MG and U251MG were broken down through a series of microarrays and gene expression. To activate HOXA9 overexpression in U87MG, the cell line was infused with Murine immature microorganism infection, the cell line was then separated in HOXA9 low versus high. Overexpression in HOXA9 as shown by Goncalves et al results in aggressiveness within the cell lines, as well as increased proliferation and invasion. ${ }^{27}$ However when HOXA9 silencing was done no proliferation was seen and there was a decrease in the size of the cell lines. It can, in this way, be recommended that HOXA9 silencing is essential in decreasing tumor size as shown in this study, in addition to current treatment modalities.

A comparison was made between healthy cells and glioma cells by costa et al, and they found no proof that healthy cells expressed HOXA9, this might paint HOXA9 as a prognostic biomarker. ${ }^{23}$ Moreover, these expressions were for the most part obvious in grade 3 to 4 tumor cells. This further lays accentuation on the idea that HOXA expressions are implicated in tumors that are dangerous and progressively intrusive, again expression was not steady in second rate tumors. Moreover, the discovery which can be utilized to foresee the evaluation of GBM relying upon expression can be utilized to decide the progress of the tumors.

A relationship between poor clinical results and HOXA9 was made by the proof Costa et al provided. ${ }^{23}$ As earlier stated, one of the fundamental expressed genes found by
Costa et al was HOXA9, the expression of this gene gave rise to low endurance rates 9 weeks when contrasted with 53 weeks of less expressed genes. ${ }^{23}$ This stresses the idea of HOXA genes, providing prognostic prediction as well as a biological marker, in this case, HOXA9. A comparison couldn't be made with expression noted during embryonic development as Costa noticed the attributes of this expression, which was portrayed as "aberrant". ${ }^{23}$ Abnormal or erroneous expression of these genes have likewise been associated with outcome prediction. Restraint of the $\mathrm{p} 13 \mathrm{k}$ pathway was advantageous in improving clinical results and lessening treatment opposition, as it switches bizarre transcription of HOXA9 expression.

The role played by signaling pathways in HOXA genes, as well as the inclusion of expression regulators, for example, RNA. This affiliation was found recently by Quaglaita et al in liver disease, they found that the genomic area of lncRNA HOTTIP (long non-coding RNA's) was personally identified with HOXA13. ${ }^{28}$ Increased expansion inside the malignant cell was seen when overexpression of lncRNA's occurred. In patients with hepatocellular carcinoma, the clinical result was predicted by lncRNA HIT (HOXA transcript prompted by TGF $\beta$ ). ${ }^{28}$ A decent handle of the different parts that add to proliferative impacts of HOXA expression will help recognize biomarkers that are explicit to glioma stages, furthermore, visualization will be improved in these patients if the dismissal of hostile to malignant growth agents is understood, ${ }^{29}$ as previously mentioned, early recognition (stage 1 ) is directly proportional to a higher level of recession success. Analysis of western smear by Duan et al., prompted discoveries of variant upregulation of HOXA9, HOXA10, HOXA11, and HOXA13 in various evaluation glioma cells (Figure 1A.) ${ }^{29}$ The job of mRNA, HOTTIP and HOXA genes were additionally researched through qPCR. It was found that increased expression is directly proportional to increasing glioblastoma grades. ${ }^{29}$ Furthermore, high-grade gliomas additionally had an increased HOTTIP transcription level (Figure 1B). HOXA13 was additionally examined to foresee clinical progression and check by and large survival, ${ }^{29}$ Using Kaplan-Maier analysis Duan et al., found a conversely corresponding connection between HOXA13 expression and overall survival, ${ }^{29}$ recommending an ascent to molecular prognostic and diagnostic tools. Although the fundamental pathology of HOXA gene in malignant growth development and progression has not been completely researched past their molecular contributions, Duan et al anyway distinguished likely genes related with HOXA13 expression in glioma cells. ${ }^{29}$ Overlap of genes were found inside the Rembrandt glioma samples and CGGA and TCGA when they were examined. To recognize the overlapping genes, an analysis was made utilizing the KEGG pathway, it was seen that genes in a pathway distinguished as a harmful one were found, the Wnt signaling pathway. This proposes TGF-b and Wnt signaling add to the abrasiveness of HOXA13 and advanced intrusiveness. Expressing HOXA13 activates genes arranged in these pathways; this was demonstrated by means of Gene set enrichment analysis, focusing on the job of signaling pathways in glioma progression.

$\mathrm{Li}$ et al gave additional proof, researched lncRNAHOTAIRM1, which was interpreted from HOXA1 
antisense. ${ }^{30}$ Expression of HOTAIRM1 by various grades of glioma cells inside A172 and U87 was contemplated, expression was likewise contrasted with healthy brain tissues. $^{30}$ They discovered HOTAIRMI expression basically in high-grade glioma and nothing in healthy tissues, proposing the role of HOXA genes/HOXA segments as valuable prognostic and diagnostic biomarkers. ${ }^{30}$ Similar outcomes were found in $\mathrm{Li}$ et al and Duan with the level of expression corresponding with grade malignancy. ${ }^{29,30}$ As expression essentially increased in grade 4 tissues. ${ }^{30}$ Levels of expression were connected with age and sex, to additionally distinguish expression as a principle threat indicator, however, no significance was found. To comprehend the impacts of HOTAIRM in glioma cells, ${ }^{30}$ diminished expressions through RNA impedance. It was uncovered by means of examining the CCK 8 and BrdU cell proliferation assay, that tumor development and proliferation was reduced inside the cells, this knockdown additionally raised apoptosis inside these cell lines. ${ }^{30} \mathrm{~A}$ decrease in proliferation and glioma cells was observed following the introduction of lentivirus and shHOTAIRM1 (RNA meddled in these cell lines, this was apparent in vivo xenograft test. Treatment obstruction can be incredibly decreased by executing this finding. ${ }^{24}$

There is a resistance to Temozolomide, a chemotherapeutic intervention, as prior expressed this is because of the basic molecular resistance in PI3-kinase interceded stem cell, which is accompanied by an overexpressed DNA repair enzyme MGMT. Prompted cytotoxicity of MGMT is important for ideal usefulness of Temozolomide, on account of decreased functional repair of the protein. ${ }^{24}$ Silencing of gene promoters, for example, HOXA genes, is one of the advantages of diminishing MGMT. HOXA genes are embroiled in differentiation meaning there is a decrease in apoptosis accordingly promoting tumor growth. ${ }^{24}$ HOXA9 and HOXA 10 were found by Gaspar et al found as the significant genes answerable for resistance built to Temozolomide owing to a great extent, their trademark of self-restoration, expression likewise decided endurance (Figure 2).

In any case, underexpression of the HOXA gene has likewise been related to poor prognosis and resistance to treatment by different studies. Se et al. keep up that after embryogenesis Homeobox genes ought to be quiet in the adult brain as their proliferative nature go about as a trigger for tumor growth ${ }^{31}$ Young-Bem Se et al asserts higher mortality is seen with underexpression of HOXA11 in GBM patients. This paper broke down five tumor tissue tests including U251, U373, and LN18 from GBM patients. HOXA11 overexpression was seen inside the phone lines, so as to knockdown HOXA11 (siHOXA11) meddling RNA tests were led on the lines through grouping focusing on, non-focused on siRNA were utilized as control. ${ }^{31}$ The utilization of Affymetrix GeneChip, investigated the adjustment in gene expression so as to identify contrast in the expression of HOXA11 knockdown and siRNA. Young-Bem Se discovered by means of the utilization of western blot that high expression of HOXA11 was directional proportional to high rates of survival, the high survival rate was $(31 \pm 15.3$ months $)$ contrasted with low survival rate $\left(18 \pm 7.3\right.$ months). ${ }^{31}$ To quantify impedance to treatment an extra in vitro examination was directed.
HOXA11 was stifled inside the cell lines of U251, U373, and LN18 the Glioma cells were then treated with radiotherapy and Temozolomide, it was discovered that inside the HOXA11 knockdown cells the anticancer factors inside the medicines declined. Focusing on HOXA11 can thusly be valuable in forestalling treatment opposition in GBM patients. $^{31}$

\section{Conclusion}

The significance of cell regulators as a method for improving results in GBM patients can't be overemphasized as confirmed by these studies. Evidence shows a decrease in multiplication for regulating HOX genes. Also imperative from these discoveries is that, either as supporters of the advancement of malignancies or as biomarkers in susceptibility to anti-cancer agents, developmental genes, for example, HOX genes might be important in carcinogenesis. This survey additionally indicated that examining regulatory mechanisms, for example, abnormally expressed HOX genes in solid tumors which are regulated by means of lincRNAs and miRNAs may give data on new focuses to compelling malignancy therapeutics.

Molecular focused treatment ought to be the focal point of future exploration as current modalities of treatment, for example, radiotherapy and chemotherapy produce helpless outcomes and abysmal rates of survival. Implementation of cell regulators as a major aspect of treatment would ultimately lead to increment effectiveness of treatment and decrease the utilization of intrusive modalities, for example, surgical procedures. The job of HOX gene expression in CSCs ought to be studied as it could give a method for structuring CSC-focused treatments, as CSCs have an impact on commencement and progression of solid tumors.

\section{Acknowledgement}

Profound appreciation to Dr. Maria Esposito for her tutelage throughout this review.

\section{Conflict of Interest}

None.

\section{References}

1. Hanif F, Muzaffar K, Perveen K, Malhi SM, Simjee SU. Glioblastoma Multiforme: A review of its epidemiology and pathogenesis through clinical presentation and treatment. Asian Pac J Cancer Prev APJCP; 2017. Jan;18(1):3-9.

DOI: 10.22034/APJCP.2017.18.1.3

2. Agnihotri S, Burrell KE, Wolf A, Jalali S, Hawkins C, Rutka JT, et al. Glioblastoma, a brief review of history, molecular genetics, animal models and novel therapeutic strategies. Arch Immunol Ther Exp (Warsz); 2013. Feb;61(1):25-41.

DOI: $10.1007 / \mathrm{s} 00005-012-0203-0$

3. Thakkar JP, Dolecek TA, Horbinski C, Ostrom QT, Lightner DD, Barnholtz-Sloan JS, et al. Epidemiologic and molecular prognostic review of glioblastoma. 
Cancer Epidemiol Biomark Prev Publ Am Assoc Cancer Res Cosponsored Am Soc Prev Oncol; 2014. Oct;23(10):1985-96. DOI: 10.1158/1055-9965.EPI-140275

4. Rock K, McArdle O, Forde P, Dunne M, Fitzpatrick D, O'Neill B, et al. A clinical review of treatment outcomes in glioblastoma multiforme--the validation in a non-trial population of the results of a randomised Phase III clinical trial: has a more radical approach improved survival? $\mathrm{Br}$ J Radiol; 2012. Sep;85(1017):e729-733. DOI: 10.1259/bjr/83796755

5. Ohgaki H, Kleihues $P$. The definition of primary and secondary glioblastoma. Clin Cancer Res Off J Am Assoc Cancer Res; 2013. Feb 15;19(4):764-72. DOI: 10.1158/1078-0432.CCR-12-3002

6. Iacob G, Dinca EB. Current data and strategy in glioblastoma multiforme. J Med Life; 2009. Dec;2(4):386-93. Available from: https://www.ncbi.nlm.nih.gov/pmc/articles/PMC30190 $11 /$

7. Ohgaki H. Epidemiology of brain tumors. Methods Mol Biol Clifton NJ. 2009;472:323-42.

DOI: 10.1007/978-1-60327-492-0_14

8. Salvati M, Frati A, Russo N, Caroli E, Polli FM, Minniti G, et al. Radiation-induced gliomas: Report of 10 cases and review of the literature. Surg Neurol. 2003; Jul;60(1):60-7; discussion 67.

DOI: $10.1016 / \mathrm{s} 0090-3019(03) 00137-\mathrm{x}$

9. Fisher JL, Schwartzbaum JA, Wrensch M, Wiemels JL. Epidemiology of brain tumors. Neurol Clin; 2007. Nov;25(4):867-90, vii. DOI: $10.1016 /$ j.ncl.2007.07.002

10. Adamson C, Kanu OO, Mehta AI, Di C, Lin N, Mattox $\mathrm{AK}$, et al. Glioblastoma multiforme: A review of where we have been and where we are going. Expert Opin Investig Drugs [Internet]; 2009 Aug [cited 2020 May 14];18(8):1061-83. Available from: https://www.tandfonline.com/doi/full/10.1517/1354378 0903052764

11. Nakada M, Kita D, Watanabe T, Hayashi Y, Teng L, Pyko IV, et al. Aberrant signaling pathways in glioma. Cancers; 2011. Aug 10;3(3):3242-78. DOI: $10.3390 /$ cancers 3033242

12. Nelson SJ, Cha S. Imaging glioblastoma multiforme. Cancer J Sudbury Mass; 2003. Apr;9(2):134-45. DOI: 10.1097/00130404-200303000-00009

13. Neurologic Manifestations of Glioblastoma Multiforme Clinical Presentation: History, Physical, Causes [Internet]. [cited 2020 May 15].

Available from:

https://emedicine.medscape.com/article/1156220clinical

14. Kesari S. Understanding glioblastoma tumor biology: the potential to improve current diagnosis and treatments. Semin Oncol; 2011. Dec;38 Suppl 4:S2-10. DOI: 10.1053/j.seminoncol.2011.09.005

15. MP C, B E, HR S, H S, J F, M H, et al. Cancer Incidence in Five Continents Volume IX [Internet]. [cited 2020 May 14]. Available from: https://publications.iarc.fr/Book-And-ReportSeries/Iarc-Scientific-Publications/Cancer-IncidenceIn-Five-Continents-Volume-IX-2007
16. Friedman HS, Kerby T, Calvert H. Temozolomide and treatment of malignant glioma. Clin Cancer Res Off $\mathbf{J}$ Am Assoc Cancer Res; 2000. Jul;6(7):2585-97. Available from: https://pubmed.ncbi.nlm.nih.gov/10914698/

17. Scott J, Tsai Y-Y, Chinnaiyan P, Yu H-HM. Effectiveness of radiotherapy for elderly patients with glioblastoma. Int J Radiat Oncol Biol Phys. 2011 Sep 1;81(1):206-10. DOI: 10.1016/j.ijrobp.2010.04.033

18. Chang JE, Khuntia D, Robins HI, Mehta MP. Radiotherapy and radiosensitizers in the treatment of glioblastoma multiforme. Clin Adv Hematol Oncol HO; 2007. Nov;5(11):894-902, 907-15. Available from: https://pubmed.ncbi.nlm.nih.gov/18185489/

19. Stupp R, Taillibert S, Kanner AA, Kesari S, Steinberg DM, Toms SA, et al. Maintenance therapy with tumortreating fields plus temozolomide vs temozolomide alone for glioblastoma: A randomized clinical trial. JAMA; 2015. Dec 15;314(23):2535-43.

DOI: $10.1001 /$ jama.2015.16669

20. Seymour T, Nowak A, Kakulas F. Targeting aggressive cancer stem cells in glioblastoma. Front Oncol; 2015;5:159. DOI: 10.3389/fonc. 2015.00159

21. Shah N, Sukumar S. The Hox genes and their roles in oncogenesis. Nat Rev Cancer; 2010. May;10(5):36171. DOI: $10.1038 / \mathrm{nrc} 2826$

22. Bhatlekar S, Fields JZ, Boman BM. HOX genes and their role in the development of human cancers. J Mol Med Berl Ger; 2014. Aug;92(8):811-23.

DOI: $10.1007 / \mathrm{s} 00109-014-1181-y$

23. Costa BM, Smith JS, Chen Y, Chen J, Phillips HS, Aldape KD, et al. Reversing HOXA9 oncogene activation by PI3K inhibition: epigenetic mechanism and prognostic significance in human glioblastoma. Cancer Res [Internet]; 2010. Jan 15 [cited 2020 May 14];70(2):453-62. Available from: https://www.ncbi.nlm.nih.gov/pmc/articles/PMC28499 35/

24. Gaspar N, Marshall L, Perryman L, Bax DA, Little SE, Viana-Pereira $M$, et al. MGMT-independent temozolomide resistance in pediatric glioblastoma cells associated with a PI3-kinase-mediated HOX/stem cell gene signature. Cancer Res; 2010. Nov 15;70(22):9243-52. DOI: 10.1158/0008-5472.CAN$10-1250$

25. Pojo M, Gonçalves CS, Xavier-Magalhães A, Oliveira AI, Gonçalves T, Correia S, et al. A transcriptomic signature mediated by HOXA9 promotes human glioblastoma initiation, aggressiveness and resistance to temozolomide. Oncotarget [Internet]; 2015 Feb 20 [cited 2020 May 15];6(10):7657-74. Available from: https://www.ncbi.nlm.nih.gov/pmc/articles/PMC44807 $07 /$

26. Neville SE, Baigent SM, Bicknell AB, Lowry PJ, Gladwell RT. Hox gene expression in adult tissues with particular reference to the adrenal gland. Endocr Res; 2002. Nov;28(4):669-73. DOI: 10.1081/erc120016984

27. Gonçalves CS, Xavier-Magalhães A, Pojo M, Oliveira AI, Correia S, Reis RM, et al. Transcriptional profiling of HOXA9-regulated genes in human glioblastoma cell models. Genomics Data [Internet]. 2015 May 19 [cited 2020 May 14];5:54-8. Available from: 
https://www.ncbi.nlm.nih.gov/pmc/articles/PMC45839 97/

28. Quagliata L, Matter MS, Piscuoglio S, Arabi L, Ruiz C, Procino A, et al. Long noncoding RNA HOTTIP/HOXA13 expression is associated with disease progression and predicts outcome in hepatocellular carcinoma patients. Hepatol Baltim Md; 2014. Mar;59(3):911-23. DOI: 10.1002/hep.26740

29. Duan R, Han L, Wang Q, Wei J, Chen L, Zhang J, et al. HOXA13 is a potential GBM diagnostic marker and promotes glioma invasion by activating the Wnt and TGF- $\beta$ pathways. Oncotarget [Internet]; 2015. Jul 31 [cited 2020 May 14];6(29):27778-93. Available from: https://www.ncbi.nlm.nih.gov/pmc/articles/PMC46950 $25 /$
30. Li Q, Dong C, Cui J, Wang Y, Hong X. Overexpressed IncRNA HOTAIRM1 promotes tumor growth and invasion through up-regulating HOXA1 and sequestering G9a/EZH2/Dnmts away from the HOXA1 gene in glioblastoma multiforme. J Exp Clin Cancer Res CR; 2018. Oct 30;37(1):265.

DOI: 10.1186/s13046-018-0941-X.

31. Se Y-B, Kim SH, Kim JY, Kim JE, Dho Y-S, Kim JW, et al. Underexpression of HOXA11 is associated with treatment resistance and poor prognosis in glioblastoma. Cancer Res Treat Off J Korean Cancer Assoc; 2017. Apr;49(2):387-98.

DOI: $10.4143 /$ crt.2016.106 